\title{
Politique Colonial D'enseignement Et Integration Scolaire Des Femmes Congolaises En Periode Coloniale Au Congo Kinshasa Exemple De Kisangani (Ex. Stanley Ville)
}

\author{
Thérèse Olonga Olulu \\ Assistante en Sciences Politiques et Administratives à \\ l’Université de Kisangani, Congo
}

doi: 10.19044/esj.2017.v13n16p157 URL:http://dx.doi.org/10.19044/esj.2017.v13n16p157

\begin{abstract}
The setting up of a teaching layman system in view of the local (work hand) colonizer of the Congo Belge, constitute the way of entry of Kisangani (former stanley city) women in order to benefit the school formation, This, however, is in spite of the traditional reticence that was observed. As for what concerns the social consideration, this opportunity seems to have promoted the man more to the woman's detriment. In fact, the documentary analysis and interview carried out with agents charged the archives of the primary and secondary education. Some women who were witness during the colonial time have helped in analysing this work. It comes out again that the minority which were considered to be the women of the city of Kisangani (former stanley city) was not neglected (disregarded). This means that some women were integrated into the school environment in the course of this period. However, in Kisangani, former Stanley city, girls' schools which existed since 1924 such as Mapendano Secondary school and Grison primary school (domestic school) were created in 1935. Later in 1954, one of them gave attention to the creation of the primary girl's schools amerced by the Mary missionary in the indigenous cities. However, these schools do not have an official objective to prepare the woman to participate in public life. This is because their objectives were not to prepare colonizer's charging, but to offer him an auxiliary staff and maneuver (housewife, PP5 and D4 instructress). The bound factors of women experiences are not to be negligent based on their school integration. Nevertheless, this formation allowed auxiliary staff to widen their field of action to the public life involvement. This also is seen as a victory on the traditional brakes.
\end{abstract}

Keywords: Educational politics, Teaching, Woman, sex, gender 


\section{Résumé}

La mise en place d'un système d'enseignement laïc en vue de la main d’œuvre locale par le colonisateur au Congo-belge a constitué la voie d’entrée pour les femmes de Kisangani (ex. Stanley ville) de bénéficier de la formation scolaire, en dépit des réticences traditionnelles observées. Concernant la considération sociale dans les faits, cette opportunité semble avoir plus promu l'homme au détriment de la femme. En effet, l'analyse documentaire et l'entretien avec les agents chargés des archives de l'enseignement primaire et secondaire et quelques femmes de l'époque coloniale nous sont utiles pour cerner l'objet du présent travail. Il ressort que la minorité peu considérée des femmes de la ville de Kisangani qui ont étudié à cette époque n'est pas à négliger. C'est-à-dire que quelques femmes ont intégré le milieu scolaire en cette période. A Kisangani, les écoles des filles sont créées depuis 1924, tels sont les cas du lycée Mapendano et de l'école primaire MGR Grison, créée en 1935. Plus tard en 1954 on assistât à la création des écoles primaires des filles assurées par les filles missionnaires de Marie dans les cités indigènes. Les facteurs liés au vécu des femmes n’est pas non plus à négliger quant à leur intégration scolaire. Néanmoins, cette formation a permis à ce personnel auxiliaire d'élargir leurs champs d'action dans la participation à la vie publique. Une triomphe sur les friens traditionnels.

Mots-cles: Politique d’enseignement, Enseignement, Femme, sexe, genre

\section{Introduction}

«C'est par l'éducation que les filles et les femmes obtiendront la liberté de faire des choix, de façonner leur avenir et d’édifier des sociétés plus inclusives et plus justes !» (UNESCO. 2011)

La mise en place d'un système d'enseignement laïc non discriminatif pour tous en vue de la main d'œuvre locale par le colonisateur en RD Congo a constitué la voie d'entrée pour les femmes de Kisangani de bénéficier de la formation scolaire, en dépit des réticences traditionnelles observées. Cette opportunité semble avoir plus promu l'homme au détriment de la femme quant à ce qui concerne la considération sociale dans les faits.

A ce sujet Kita K. M. (1960) affirme que le cas particulier de l'Afrique fournit une raison supplémentaire à l'étude de l'enseignement colonial. En effet, en étudiant les buts réels visés de cet enseignement colonial, les objectifs officiels proclamés et en confrontant à la fois aux moyens mis en œuvre et aux résultats obtenus, le chercheur se donne une double possibilité.

D’une part, il peut mettre en évidence la part de mystification qui à bien des égards et pendant longtemps, a emportée l'enseignement colonial. 
D’autre part, cela lui permet d'analyser objectivement les conséquences multiples de cet enseignement sur les bénéficiaires et sur la société. Nous ne pouvons oublier, en effet, que ceux qui ont bénéficié de cet enseignement colonial constituent une minorité, certes, mais une minorité politiquement importante.

Minani R.B., cité par Tshibilondi (2010) affirme que pour mieux comprendre la situation de la femme congolaise, il faut examiner sa situation dans le domaine de l'éducation.

Un recul dans l'histoire coloniale de la scolarisation de la femme congolaise est donc indispensable pour faire une lecture de son intégration scolaire.

La plupart des écrits en matière de la situation de la femme congolaise condamne le colonisateur de l'impréparation de la femme à l'accès aux postes de décision. Cette prise de position peut être relativisée. Car la cote-ouest par laquelle les colonisateurs sont entrés a bénéficié de la formation des jeunes filles. Les premières sœurs de la charité de Gared, arrivées vers les année 1897, trois ans après le père Cambier, premières religieuses et femmes européennes à pénétrer à l'intérieur de cet immense pays, l'éducation des jeunes filles et le développement des femmes sont pris en charge » L’Archidiocèse de Kananga (1982) dans la cote-ouest.

Pour ce qui est de la ville de Kisangani, c'est à la naissance des quelques écoles des filles et des écoles mixtes que les femmes de Stanley ville ont intégré le monde scolaire. Cette intégration a été aussi favorisée par leurs situations familiales, leur parcours et l'esprit de la concurrence.

Le présent travail, se propose d'analyser la politique coloniale d'enseignement et l'intégration de la femme de Kisangani (Ex. Stanley ville) en milieu scolaire. Il s’agit de répondre à la question suivante : comment la femme de Kisangani a-t-elle intégré le monde scolaire face à la politique coloniale d'enseignement, en dépit des pratiques traditionnelles ?

Nous nous proposons ainsi de vérifier l’hypothèse selon laquelle: La politique coloniale belge d'enseignement aurait favorisé l'intégration scolaire de la femme grâce à la formation de quelques femmes malgré les réticences traditionnelles et les préjugés suite à la nécessité de l'existence d'une politique d'enseignement pour tous en vue de la main d'œuvre locale.

L’objectif de la présente étude est de déterminer dans la politique coloniale belge en matière de l'enseignement et les causes qui sont à la base de l’intégration scolaire de la femme de Kisangani (Ex. Stanley ville).

La présente analyse porte sur trois points essentiels dont la première porte sur la clarification conceptuelle, Le deuxième sur Les déclencheurs de la scolarisation des femmes dans politique coloniale belge en matière de l'enseignement au Congo belge (ex. Stanley ville) dans lequel nous passons en revue, l'histoire de l'enseignement colonial belge et les 
disposition réservées aux filles à cette époque, et le troisième point enfin porte sur les femmes de Kisangani (Ex. Stanley ville) en milieu scolaire.

\section{Methodologie}

Pour collecter les données requises pour cette étude, nous avons utilisé les techniques suivantes: La technique documentaire qui nous a servi à chercher, identifier et trouver des documents relatifs à ce sujet de recherche; l'interview et l'entretien libre nous ont permis d'échanger directement avec nos enquêtés.

Dans le cas d’espèce, il s’agit des anciennes élèves des écoles existantes à l'époque coloniale à Kisangani. Les responsables de ces écoles, les agents de la Division Provinciale de l'Enseignement Primaire et Secondaires. Le récit de vie (ou la biographie genrée (Welepele, 2013)) nous a été utile pour Causer avec les enquêté(e)s afin de les amener à relater en profondeur leur expérience de vie ou la manière dont ils ont vécu ou vivent la domination au quotidien ne saurait être exploré aux cours d'un simple procédé interactif comme celui de l'entretien. Cela demande la mise au point d'une technique narrative. Voilà pourquoi le récit de vie ou la biographie (genrée) des acteurs/actrices qui ont été d’ultime recours dans le cadre de ce travail.

Quant à l'échantillonnage nous avons opté de consulter les femmes formées pendant l'époque coloniale, quelques autorités coloniales et les témoins de cette époque.

\section{Clarification conceptuelle}

La présente étude inspire la clarification préalable des concepts politique publique, enseignement, femme, sexe et genre.

\section{Politique publique}

Il existe une multitude des définitions possibles des politiques publiques. L’une des plus simples consiste à considérer que les politiques publiques sont tout ce qu'un gouvernement choisi de faire ou de ne pas faire (Nay, 2014).

Cette notion recouvre plusieurs sens selon l'usage qui en est fait. Selon J.C. Thoenig, une politique publique est un programme d'action propre à une ou plusieurs autorités publiques ou gouvernementales. Pour Yves Meny et J.C. Thoenig (1989) une politique publique est le produit d'une autorité investie des puissances publiques et de légitimité gouvernementale, autorité considérée comme organe public régulier.

Pour ce qui nous concerne, nous considérons une politique publique comme des réponses explicites ou implicites apportées par des autorités publiques aux malaises sociaux à travers leurs programmes d'action. Pour le 
cas d’espèce, il s’agit de l’intégration scolaire de la femme de Kisangani (ex : Stanley-ville) en période coloniale.

\section{Enseignement}

La philosophie de l’éducation, puis le courant anglo-saxon de l'étude de l'enseignement (study of teaching), ont depuis longtemps travaillé sur la notion d'activité d'enseignement. Plus récemment, les champs de recherche de la psychologie du développement et de l'éthologie ont recueilli un ensemble de données sur cette activité, en s’éloignant de l'enseignement en milieu strictement scolaire (Philippe Dessus, 2008).

Si l'activité d'enseignement a donné lieu à un très grand nombre de travaux, il existe peu de synthèses tirant parti de ces travaux pour en proposer une définition large, qui puisse s’appliquer à la fois aux domaines scolaire et non scolaire.

Toutefois, la plupart des résultats des recherches dans ces différentes disciplines sont assez en accord sur la finalité de l'enseignement : la transmission culturelle d'humains experts au naïfs, ou, comme le formulent Premack « [...] réduire la disparité entre les actes du novice qui sont observés et les standards internes du pédagogue » (Philippe Dessus, 2008).

Le mot éducation a été parfois employé dans un sens très étendu pour désigner l'ensemble des influences que la nature ou les autres hommes peuvent exercer soit sur notre intelligence, soit sur notre volonté. Elle comprend, dit Stuart Mill, « tout ce que nous faisons par nous-mêmes et tout ce que les autres font pour nous dans le but de nous rapprocher de la perfection de notre nature. Dans son acception la plus large, elle comprend même les effets indirects produits sur le caractère et sur les facultés de l'homme par des choses dont le but est tout différent : par les lois, par les formes du gouvernement, les arts industriels, et même encore par des faits physiques, indépendants de la volonté de l'homme, tels que le climat, le sol et la position locale» (Philippe Dessus, 1922).

Mais cette définition comprend des faits tout à fait disparates et que l'on ne peut réunir sous un même vocable sans s'exposer à des confusions. L'action des choses sur les hommes est très différente, par ses procédés et ses résultats, de celle qui vient des hommes eux-mêmes; et l'action des contemporains sur leurs contemporains diffère de celle que les adultes exercent sur les plus jeunes. C'est cette dernière seule qui nous intéresse ici et, par conséquent, c'est à elle qu'il convient de réserver le mot éducation. Suivant Kant (2013), « le but de l'éducation est de développer dans chaque individu toute la perfection dont il est susceptible »

L'enseignement peut s'entendre comme une suite logique des connaissances construites les unes sur les autres et qui viennent meubler l'esprit de l'homme. 
Par enseignement, nous entendons le moyen le plus important par lequel la femme congolaise en générale et celle de Kisangani en particulier devrait être préparé à sa participation à la vie politique. Le taux des femmes admises en milieu scolaires, les modalités d'admission de la femme et le type d’enseignement donné à la femme à une conséquence sur sa promotion politique.

\section{Femme, sexe et genren (Welepele, 2013)}

Définie comme un être humain de sexe féminin, la femme est selon Larousse (2010): un adulte de sexe féminin ; comme épouse. Alors qu’il considère l'homme comme un être humain de sexe masculin, adulte de sexe masculin. Ces deux définitions considèrent le «sexe biologique » comme élément constitutif et distinctif entre les hommes et les femmes.

La plupart des définitions qui tentent de définir l’homme et la femme insistent sur les caractères biologiques et physiologiques, c'est-à-dire elles essaient de saisir et de comprendre l'homme et la femme par leur nature respective. Il s’agit là des considérations essentialistes, naturalistes qui mettent le marqueur de “'sexe”' au centre de la différenciation entre l'homme et la femme.

Cependant, l'on observe que, ces définitions ne font pas cas des droits sociaux de la femme. Depuis longtemps en effet, la naturalisation de la catégorie « femme » a été utilisée comme prétexte pour justifier l’oppression, l'exploitation ou la domination des femmes par les hommes. La différence entre les sexes était le fondement de la discrimination de femmes et de leur « invisibilité » dans l'espace public. Face à cette tendance, les féministes en sont parvenu(e)s à forger et introduire dans le débat public le concept de «Gender » ou de "genre, rapports sociaux de sexe » dans le but de démontrer le caractère socialement / culturellement construit de la différence entre les sexes (Hirata et al., 2000).

Dans son usage le plus ancien, «genre » était synonyme de « femme » lorsqu'on parlait de genre, on avait simplement l'idée de femme ou du genre féminin. Des livres et articles de toutes sortes, qui avaient comme sujet l'histoire des femmes ont substitué dans leurs titres le terme de « genre » à celui de «femmes ». Mais que signifie alors genre ? Genre en tant que substitut pour «femmes » est également utilisé pour suggérer que l'information au sujet des femmes est également informations sur les hommes, que l'un implique l'étude de l'autre ; cet usage insiste sur le fait que le monde des femmes fait partie du monde des hommes, qu'il est créé dans et par ce monde.

En réalité, l'être humain comporte deux dimensions : l'inné et l'acquis. BEAUVOIR DE S., dans «le deuxième sexe » tome 2 a écrit: 'être'" une femme ou un homme, ''avoir'" un sexe masculin ou 
féminin représente d'avantage l'effet des processus sociaux qu'un état naturel antérieur à tout. Les êtres sont avant tout des humains avant de paraître femmes ou hommes. (...) On ne nait pas femme, on le devient. Aucun destin biologique, psychique, économique ne définit la figure que revêt au sein de la société la femelle humaine ; c'est l'ensemble de la civilisation qui élabore ce produit intermédiaire entre le mâle et le castrat qu'on qualifie de féminin (Beauvoir, 1949).

Cette idée marque un tournant décisif dans la pensée féministe en ce sens qu'elle met en évidence les différences sexuelles socialement, culturellement construites. On passe ici du sexe biologique au sexe social.

Pour le cas d'espèce, il s'agit bien de sexe social, car l'accès à l'enseignement est ouvert à tous (les hommes comme les femmes) par le colonisateur, sans tenir compte de sexe biologique. Mais ce sont les habitudes sexuées de la culture et des traditions qui font susciter le débat de l'accès ou non du sexe féminin à la formation scolaire.

\section{Les déclencheurs de la scolarisation des femmes dans politique coloniale belge en matière de l'enseignement au Congo belge (ex. Stanley ville).}

Les déclencheurs de la scolarisation des femmes en période coloniale sont liés à la politique coloniale d'enseignement. Raison pour laquelle dans point n'est besoin d'analyser cette politique d'abord d'une manière générale, mais de cadrer l'analyse de la politique coloniale d'enseignement de manière spécifique pour les filles.

Depuis l'Etat Indépendant du Congo, l'enseignement colonial au Congo Belge a été régi par des Conventions entre l'Administration coloniale et les Missions chrétiennes. L'intégration scolaire des femmes en cette période est liée à l'évolution historique du moment.

En 1906, le Saint Siège et l'Etat Indépendant du Congo signaient une Convention, qui formulait les grands principes de l'organisation scolaire dans la Colonie. Peu après la reprise de l’Etat Indépendant du Congo par la Belgique en 1908, on commença à élaborer un texte d'organisation de l'enseignement libre des Missions Nationales qui sera adopté en 1929, temps mort créé à cause de la première guerre mondiale.

Ce programme a subi plusieurs modifications dans le temps (Organisation de l'Enseignement libre au Congo Belge et au Ruanda-Urundi, 1958), selon qu'il passait de l'égide des missionnaires à celui de l'Etat et vice versa. Il avait comme mission première de répondre aux désirs d'évangélisation des missionnaires, notamment catholiques (Organisation de l'Enseignement libre au Congo Belge et au Ruanda-Urundi , 1929), et aux besoins de la métropole de former des cadres d'exécution au service de l'économie et de l'Administration Coloniale. 
Il convient de signaler que, ces programmes d'enseignement étaient non discriminatoires pour les hommes comme les femmes et les religieux comme les laïcs étant donné le silence des documents juridiques à cet effet. les réticences traditionnelles, la sous-estimation de soi de la part des femmes ont bloqué la voix d'accès à l'école.

Le régime de l'enseignement est quand même double au Congo belge. C'est-à-dire, d'une part les écoles conventionnées qui font le mixage des enseignements religieux et l'activité scolaire selon l'Etat. D'autre part par contre, il y a des écoles officielles qui ne font que l'activité scolaire selon l'Etat.

\section{Une scolarisation spécifique pour les filles}

D’après les dispositions juridiques de l'époque, les filles doivent être associées à l'éducation agricole et être enrôlées dans une section ménagèreagricole. Le programme de celle-ci comportera tous les travaux agricoles et domestiques, les industries domestiques locales ainsi que les soins aux malades et aux blessés.

Les travaux des sections professionnelles devront être adaptés aux besoins des industries régionales. Il conviendra de les préciser avec le concours des chefs d'industries de la région. Là où il n'existe pas encore de grande industrie, la maçonnerie, la charpenterie, la menuiserie et l'ébénisterie enseignées en vue de la diffusion de méthodes de travail plus rationnelle occuperont la première place après l'agriculture.

Là où les travailleurs du métal ne peuvent pas encore trouver à s'employer, la forge, la serrurerie, la fonderie, le travail du cuivre pourraient néanmoins former une section d'enseignement à raison de l'importance fondamentale de ces métiers comme facteurs d'éducation et de progrès et aussi à raison de leur utilité pour l'avenir.

La valeur éducative des écoles professionnelles se trouve naturellement accrue lorsqu'elles font partie d'un groupe scolaire.

\section{Politique coloniale d'enseignement pour filles}

Jadis, en Afrique, avant l'arrivée du colonisateur, l'éducation et l'instruction étaient données sous forme d'initiation. Les dépositaires du savoir ancestral étaient les adultes/ainés, qui par leurs sagesse et intelligence, formaient les jeunes/cadet à la vie, à la sexualité et à la connaissance de l'univers. Cet apprentissage de la connaissance était réalisé selon les classes d'âge et de manière séparée entre les garons et les filles. Mais lorsque vint la colonisation, les structures de l'enseignement traditionnelles connurent des profondes mutations suite à l'introduction de l'école coloniale moderne (Welepele, 2013). 


\section{Enseignement du premier degré pour filles}

Il convient de noter que, ces écoles n’avaient pas officiellement l'objectif de préparer la femme à participer à la vie politique, car leur objectifs n’étaient pas de préparer la relève du colonisateur, mais de lui offrir un personnel auxiliaire et des manœuvre (ménagère, monitrice PP5 et D4). C'est pourquoi en cette période, l'enseignement primaire pour jeune fille diffère quelque peu de celui des garçons.

Le programme du premier degré est identique à celui des garçons ; seuls diffèrent les travaux agricoles auxquels on ajoute la couture et les travaux domestiques.

L'adoption d'un programme commun pour garçons et pour filles se justifie du fait que la plupart des écoles de village étaient mixtes. Au surplus, le programme du premier degré est un programme du dégrossissement dont le développement modeste accessible aux filles aussi bien qu'aux garçons.

\section{Enseignement du deuxième degré pour fille}

Contrairement à l'enseignement pour garçon, le second degré pour filles n’est pas dédoublé : c’est un degré unique dont l’organisation est exclusivement étudiée en vue de « l'éducation de masse ».

Ces responsables considèrent que cette éducation doit se contenter de former de «bonnes épouses et bonnes mères »; d'où l'importance des branche telles que l'agriculture, la cuisine, la lessive, le repassage, la couture, la puériculture, l’hygiène, l’entretien de la maison, etc.

Le deuxième degré pour fille s'apparente au deuxième degré ordinaire pour garçons; toutefois, les mathématiques, la géographie et certains thèmes de causeries générales, perdent considérablement de leur importance ou disparaissent du programme, au profit des branches pratiques précitées. Le deuxième degré pour fille dure trois ans. Ainsi l'enseignement primaire pour filles, comme l'enseignement primaire ordinaire pour garçons, est d'une durée de cinq ans.

Toutefois, une $6^{\text {ième }}$ année préparatoire est créée au bénéfice des jeunes filles qui étaient sélectionnées au terme de l’enseignement primaire en vue de l'école moyenne-ménagère.

Cette $6^{\text {ième }}$ préparatoire peut être complétée d’un $7^{\text {ième }}$ préparatoire si les élèves admises sont trop jeunes dans le but de combler les lacunes de l'enseignement primaire en dotant les élèves, d'un bagage suffisamment solide de connaissances générales pour leur permettre de s’assimiler avec fruit un enseignement moyen relativement important.

Le programme de la $6^{\text {ième }}$ préparatoire insiste surtout sur l'approfondissement des cours de français et de mathématiques, cherchant ainsi à se rapprocher du niveau atteint en ces deux disciplines par le $2^{\text {ième }}$ degré ordinaire pour garçons. 


\section{Enseignement post primaire pour fille: blocage idéologique et préjugés}

Alors qu'en ce qui concerne les garçons, l’école ouvre la voie à l'enseignement secondaire et trace des perspectives d'un enseignement supérieur et universitaire, la réforme de 1948 croit bon limiter la portée de l'enseignement pour filles. Le programme de l'école primaire de celles-ci est moins développé que celui des garçons; leur $6^{\text {ieme }}$ année préparatoire équivaut à peine à la $3^{\text {ième }}$ année du $2^{\text {ième }}$ degré de garçon. Au-delà de l'école primaire, seule l’école moyenne-ménagère offre le niveau le plus élevé proposé aux filles.

Selon les dispositions légales, les responsables coloniaux se disaient conscients de l'importance que revêt, dans un plan d'action civilisatrice par l'école, la formation de l’élément féminin indigène.

D’après ces responsables, « le relèvement moral et intellectuel » se poursuive les enseignements à la même cadence chez les garçons comme chez les filles. Pourtant ce pieux souhait ne passe pas dans la réalité.

La non application de ce souhait s'explique par le fait que c'est un idéal qui se trouve freiné pour plusieurs raisons liées aux circonstances défavorables à l'éducation des filles : organisation sociale indigne, atavisme de servitude qui pèse sur la femme noire, réceptivité intellectuelle généralement moindre chez les filles que chez les garçons, préjugés ou opposition intéressée du milieu familial. Nous ne pouvons songer à développer l'enseignement des filles, au même rythme, ni sur le plan aussi généralisé. Ni enfin selon le programme aussi complet que pour les garçons (Kita Kyankenge, 1982).

\section{Les filles en milieux scolaires dans la ville de Kisangani pendant la période coloniale (Stanley ville)}

Comme nous l'avons signalé avant, plusieurs chercheurs minimisent les efforts menés par les colonisateurs pour la scolarisation des jeunes filles, alors que l'histoire nous renseigne que les premières écoles des filles ont été créées dès 1924. Tel est le cas des écoles des filles de Boma.

Pour la ville de Kisangani, nous pouvons citer le lycée Mapendano qui fut créée en 1924.

En effet, sur l'initiative de MGR Grison, les frères maristes durent déménager et laissèrent la gestion de l’école primaire qu'ils dirigeaient entre les mains des sœurs franciscaines. Quelques temps après en 1935, ce fut l'inauguration de l'école ménagère. Plus tard en 1954 on assistât à la création des écoles primaires des filles assurées par les filles missionnaires de marie dans les cités indigènes.

Néanmoins, cette formation a permis à ce personnel auxiliaire d’élargir leurs champs d'action dans la participation à la vie publique. 
En effet, grâce à la pression nationaliste de l'indépendance, le colonisateur et les religieux étaient obligé de préparer ce personnel à la relève. Raison pour la quelle, à la veille de l'indépendance, ce personnel a pour la plus part assuré la gestion de la chose publique, tel est le cas de madame Mayani Lokema, ancienne élève du Lycée Mapendano en 1935, qui devint bourgmestre de la commune Makiso de 1968 à 1971.

\section{Les écoles pour filles}

En effet, les écoles ménagères, écoles des moniteurs et les écoles des sages-femmes étaient ouvertes aux filles. Ces écoles s'arrêtaient au niveau inférieur ; c'est-à-dire jusqu'à deuxième année dans les écoles ménagères et des sages-femmes; et jusqu'à quatrième année dans les écoles des moniteurs. Les monitrices ne pouvaient enseigner que jusqu'à quatrième année primaire.

Les filles de Kisangani qui voulait poursuivre les études jusqu'à obtenir le diplôme étaient envoyés soit à Lubumbashi ou à Bafwabaka.

En dépit de la politique coloniale belge d'enseignement, il faut noter que les mœurs interdisaient aux femmes de pousser les études. C'est la raison pour laquelle les études post-primaires ne recevaient que $35 \%$ des femmes. Et seuls les évolués avaient le droit d'envoyer leurs enfants à l'école.

De ce fait, selon les informations nous livrées par la Division éducationnelle de la Tshopo, quatre écoles existaient avant l'indépendance. Il s’agit de l'école Primaire Ketele, le lycée Mapendano, l'école Primaire Monseigneur Grison et l'école Ménagère de Lubunga.

Pour des raisons de manque d'archive dans les sites de recherches, seuls le lycée Mapendano et E.P Msgr Grison nous ont servi à la récolte des données quoique les effectifs des filles aient été disparu dans le mouvement de guerre.

\section{E.P Msgr Grison}

\section{Fondation (Archives de l'école Primaire MSGR Grison, 2015)}

L'ouverture de l'EP Msgr Grison a eu lieu le 02 février 1898, avec 4 élèves à la mission Sain Gabriel par Msgr Grison alors père Grison, prêtre de la congrégation des prêtres Sacré-Cœur de Jésus. L'école était au départ, une école d'alphabétisation pour la formation des catéchistes.

\section{Lieu et dénomination de l'école}

A sa fondation, l'école fonctionnait au sein même de la mission sain Gabriel. Devenue une école d'application plus tard, elle fut transférée au bâtiment abritant l'école des moniteurs où fonctionne l'actuel lycée Anuarite, avant l'occupation définitive de son propre bâtiment érigé entre la mission Saint Gabriel et le lycée Anuarite. 
L'école a connu respectivement les dénominations ci-après :

L'école primaire Saint Gabriel dès la fondation à 1972

L'école primaire mixte de la cité Kalindula de 1972 à 1974 ;

L'école primaire Ngenengene de 974 à nos jours.

Cette dernière dénomination provient du nom ruisselais qui prend sa source à côté de l'aérodrome de simisimi et qui coule à côté de l'école vers la mission Saint Gabriel.

3.

Fonctionnement

Jadis, elle fonctionnait comme cercle scolaire avec ses succursales couvrant ainsi les trois quarts de la paroisse. Ses succursales de Babusoko, Babula, Bagbangama, Yelenge et Yaoseko du total de 33 classes permirent aux enfants de ces milieux reculés de s'instruire comme leurs collègues du central. Mais, les travaux de Nganda et tout récemment l'avènement du SECOPE ont fait que l'école primaire NgeneNgene ne garde ce jour que 14 classes au chef-lieu de la paroisse.

Malgré cette diminution, l'école est comptée parmi les grandes écoles de la ville de Kisangani, cela grace au rendement et l'encadrement des élèves.

L'école primaire NgeneNgene a grandement contribué dans la formation des enfants et des jeunes de la ville de Kisangani en particulier et de la province en général.

4.

Type de formation

Au début l'école était seulement pour les garçons. Elle est devenue mixte en 1955. Ces filles ne pouvaient étudier que la première et la deuxième année. Elles allaient en suite au lycée MAPENDANO (école des sœurs franciscaine de marie) pour apprendre à devenir sage-femme, infirmière, enseignante et couturière. Les garçons partaient dans l'école normale après avoir terminé la sixième année primaire.

\section{Lycée Mapendano (Archives du Lycée Techniques MAPENDANO, 2016)}

1.

Situation géographique

Situé sur l'avenue KASSANDA au quartier des musiciens, le lycée technique MAPENDANO trouve son origine dans la première école primaire pour fille de la ville de Kisangani.

2.

Dénominations

1924 : école primaire pour fille de Kisangani ;

1954 : Institut Virgo Immaculata

1975 : Lycée YA VISIMA

1988 : Lycée Technique MAPENDANO

3.

Aperçu historique et Type de formation 
A la demande de MGR GRISON, l’école fut créée le 03 juillet $1924^{2}$ par les franciscaines missionnaire de marie arrivées en 1900 à la mission saint Gabriel, dans les locaux occupés précédemment par les frères maristes à côté de la cathédrale notre dame de très Saint Rosaire (Actuelle Banque Centrale du Congo)

En 1935, est créé la section ménagère post-primaire faisant suite aux cinq années primaires.

1952, ouverture de la sixième primaire ;

1953, création de l'école des monitrices (3 ans -D3) ;

1954 :

ménagère ; à la même année,

école ménagère poste-primaire devient école moyenne

- $\quad$ déménagement de l'école des filles des bâtiments situés près de la cathédrale au quartier des musiciens encore inachevé.

Création de la section EAP (Ecole d'apprentissage Professionnel, 2 ans) formant les enseignants pour le premier degré primaire. Cette section était transitoire et a été créée par l'administration coloniale devant l'afflux inattendu des écoliers.

- $\quad$ Création de l'école ménagère péri-primaire pour les filles trop âgées, pour commencer le primaire, l’enfant y était presqu'individualisé. Cette section disparaitra d'elle-même quelques années plus tard.

1967 :

la section vendeuse est impose par 'UNESCO comme condition à la continuation de la section moyenne ménagère. Elle disparaitra 4 ans ou 5 ans plus tard.

- $\quad$ Création du cycle d'orientation selon la réforme scolaire.

1969, ouverture du premier cycle long : humanité pélagique et d'un cycle court ; commercial.

1971, la section moyenne ménagère est remplacée progressivement par le cycle court professionnel, coupe-couture

1976, début des autres cycles longs: les humanités commerciales et administratives remplaçant le cycle court commercial et les humanités techniques qui débutent progressivement à côté du cycle court.

1978, scission de l'école primaire en deux : LYMA I et LYMA II ; ayant chacune sa propre direction et 22 salles de classe.

1980, la direction du lycée est confié aux diocésains et est divisé en deux préfectures : PEDACOM et PROTECHNIQUE.

1987-1991, suppression progressive de cycle court pédagogique.

1988, le Secoper fusionne toute les sections secondaires à une seule direction

\footnotetext{
${ }^{2}$ Notre entretien ave Maman MARIE KIMOKTO et DJAFARI anciennes élèves et enseignantes au Lysée technique MAPENDANO
} 
1989, A la demande se Son Excellence MSGR Laurent MOSENGWO, les franciscaines missionnaires de marie reprennent la direction du lycée.

1993, création de la section secrétariat (cycle court)

1996, le Secoper accorde 3 directions des études reparties de la manière suivante :

1. Humanités pédagogiques et commerciales ;

2. Humanités technique et secrétariat

$3 . \quad$ Cycle infirmière des humanités

1995-1999, suppression progressive du cycle court professionnel et début du CAP ouvert aux jeunes filles inaptes à l'enseignement secondaire pour quelques motifs que ce soit. Les élèves y apprennent la couture, les cours ménagers et le Français.

2000, début du cours d'informatique pour la section de secrétariat.

\section{Le vécu des femmes de Kisangani (ex stanley ville) intégrées en milieu scolaire}

Les histoires de vie, d'après Wright M. (1988) sont élaborées dans une perspective principalement ethnographique ou, comme dans un procès, pour apporter un élément de preuve, ou encore, à la façon de la nouvelle histoire sociale, pour illustrer les expériences et la conscience, communes ou plus rares, des gens ordinaires.

A partir de leurs récits de vie, il y a lieu de distinguer deux types des réalités dans le vécu des femmes ayant intégré le milieu scolaire en période coloniale. Il s’agit des réalités transversales et spécifiques.

\section{Les réalités transversales}

Les réalités transversales sont celles vécues en commun par ces femmes sur le plan socioculturel. A cause de nos coutumes, traditions et préjugés et autres, la fille a toujours été l'objet de discrimination, de marginalisation ou de rejet (Kasongo, 2014).

Ce qui crée de sa part, la sous-estimation de soi. Raison pour laquelle, quoi que les écoles existaient, les femmes de l'époque coloniale par le souci de ne pas être considéré comme un égal à l'homme qui doit étudier pour garantir la survie de sa famille, les femmes ainsi que leurs parents ou tuteurs ont très timidement adhéré à l'idée de la scolarisation des filles.

Les filles qui acceptaient d'étudier en cette période étaient considérées comme des garces qui n'ont pas d'ambition de se marier un jour.

En dehors de cette situation, il y a aussi la politique de vie en ville qui limitait la présence des filles dans les centres où se trouvaient les écoles et les centres de formation professionnelles. 
Le mariage constituait en soi une raison noble de ne pas étudier ou de s'arrêter à un niveau inférieur à fin de ne pas pousser les hommes au complexe.

Cependant, il y a aussi la féminité concurrentielle des femmes qui pouvaient créer l'émulation lorsqu'une femme qui a étudié est primée ou bénéficie des certains privilèges comme devenir monitrice, serveuse ou accoucheuse dans des institutions gérées par les blancs.

Par-dessus toutes ces réalités, la création des écoles des filles, la mise en place de la possibilité des filles d'étudié a favorisé l'entretien de cette émulation.

\section{Les réalités spécifiques}

Pour préserver l'anonymat garanti auprès de nos enquêtés, il convient pour nous de citer tous simplement ces réalités spécifiques. Ces réalités sont :

- L'appartenance à une famille des évolués : les filles issues des familles des évolués avaient le privilège d'intégrer le milieu scolaire à cause de l'ambiance amicale avec les enfants des blancs ;

- Le mariage à un évolué : les jeunes filles qui se sont mariés à des évolués, pour la plupart des hommes venu des Boma pour travailler à Stanley-ville ont eu le gout de la scolarisation et supportés par leurs maris ;

- $\quad$ La vie en ville ou un centre où se trouve l'école : le fait d'être proche de l'école où étudiaient les filles a aussi permis à certaines femmes de fréquenter le milieu scolaire ;

- La faveur des parents: certains parents qui n’étaient pas liés à la coutume et aux traditions ont encouragé leurs filles à étudier ;

- $\quad$ Etre seule (fille) né dans le mariage de ses parents : lors qu'on n’avait qu'un seul enfant de sexe féminin, c'est facile de la faire scolarisé par ce qu'on n'avait pas de garçons.

- Le rejet social : les filles moins considérés dans la société à cause de leurs situations étaient soumises à des traitements discriminatoires exagérés, d'où elles se refugiaient dans des écoles professionnelles.

\section{Conclusion}

A la fin de cette politique colonial d'enseignement et intégration scolaire des femmes congolaises en période coloniale au congo kinshasa. Exemple de kisangani (ex. stanley ville), il convient de noter que les femmes de Kisangani (Stanley ville) ont bénéficié d'une formation scolaire grâce à la mise en place d'un système d'enseignement vue de la main d'œuvre locale.

L'existence d'un double régime de l'enseignement au Congo belge. C'est-à-dire, d'une part les écoles conventionnées qui font le mixage des enseignements religieux et l'activité scolaire selon l'Etat et d'autre part par 
contre, il y a des écoles officielles qui ne font que l'activité scolaire selon l'Etat, est une diversité qui ouvre plusieurs horizons aux femmes de l'époque tout en garantissant une liberté de choix.

Pour la ville de Kisangani (Stanley ville), les femmes ont intégré l'enseignement scolaire depuis 1898, tel est le cas de l'EP Msgr Grison, plusieurs autres écoles officielles ou conventionnées se sont créées en suite, dont nous avons pris un exemple, celui du lycée Mapendano qui fut créée en 1924.

Bien que la politique coloniale à travers la création des écoles ait fourni une base d'intégration scolaire, cette formation des ménagères, couturières et monitrices était quand même loin d'ouvrir l'esprit créatif.

Il y a lieu de dire donc que, malgré la réticence traditionnelle, les femmes de Kisangani (Stanley ville) ont intégré la scolarisation grâce à la nécessité d'une main d'œuvre locale et la création des écoles agricoles, ménagères et des formations professionnelles. Par ailleurs, les femmes de l'époque ont intégré le milieu scolaire grâce à d'autres réalités socioculturelles transversales et spécifiques liées à leur vécu.

\section{References:}

1. Archives de l'école Primaire MSGR Grison, Novembre 2015

2. Archives du Lycée Techniques MAPENDANO, Janvier 2016

3. Beauvoir DE S. (1949). «La construction sociale des catégories de sexe », in Beauvoir DE S., Le deuxième sexe, tome 2. L'expérience vécue, Gallimard, Paris, 1949

4. Bindungwa, E.M. (2008). Comment élaborer un travail de fin de cycle ? Contenu et étapes, Médias Paul, Kinshasa.

5. Bumba Monga Ngoy et al. (2013). La politique congolaise de l'éducation : des origines à nos jours, éd. Feu torrent, Kinshasa.

6. Convention Etat Indépendant et le Saint-Siège (1906).

7. Emile Durkheim (1922). Éducation et sociologie, édition électronique réalisée à partir du livre d'Émile Durkheim, Édition Microsoft Word 2001 pour Macintosh. $\mathrm{P} 1$

8. Hirata et al. (2000). Dictionnaire critique du féminisme, PUF, Paris.

9. Jean Luc Ernest (2014). Enseignement, in http: //www. stanleyville.be /enseignement.html,_consulté le 03 Octobre.

10. Kasongo K. (2014). Politique de réhabilitation du système éducatif en République démocratique du Congo "essai d'évaluation de l'enseignement de base de 1997 à 2013, Thèse de doctorat en SPA, UNIKIN.

11. Kita kyankenge M. (1982). Colonisation et enseignement. Cas du zaïre avant 1960, éd. CERUKI, Bukavu. 
12. Larousse illustré (éd) (2010). Notre entretien ave Maman MARIE KIMOKTO et DJAFARI anciennes élèves et enseignantes au Lysée technique MAPENDANO.

13. Mopondi bende kombumbu (2014). Des objectifs de l'enseignement à la formation des enseignants En République démocratique du Congo, U.P.N.-Kinshasa inhttp://www.congoforum.be/upldocs/ , le $01 / 10 / 2014$

14. Nay O. (2014). Lexique des sciences politiques vie et institutions politiques, $3^{\text {ième }}$ éd dalloz, Paris.

15. Organisation de l'Enseignement libre au Congo Belge et au RuandaUrundi (1929). avec le concours des Sociétés de Missions nationales, Dison-Verviers.

16. Organisation de l'Enseignement libre subsidié pour indigènes avec le concours des Sociétés de Missions chrétiennes (1948). Dispositions générales, Léopoldville.

17. Philippe Dessus (2008). Qu'est-ce que l'enseignement ? Quelques conditions nécessaires et suffisantes de cette activité, In Revue Française de Pédagogie, 2008

18. Philippe Dessus (2008). Qu'est-ce que l'enseignement ? Revue Française de Pédagogie, INRP.

19. Scott J. «Genre: une catégorie utile d'analyse historique », in BISILLIAT J et VERSCHUUR C.

20. Thoenig J.C. (2011). L'analyse des politiques publiques, in Grawitz Madeleine et LECA.J (sdir), traité des sciences politiques, Tome 4 ; politiques publiques, Paris, PUF, 1985. UNESCO, «Une vie meilleure, un avenir meilleur!».

21. Tshibilondi, N. (2010). Statut de la femme congolaise de l'indépendance à nos jour (1960-2010), Centre d'Études Africaines et de Recherches Interculturelles CEAF\&RI, Communication à Ebène Plus Liège, 19 juin.

22. Welepele, E.C (2013). construction de rapport de genre et représentation politique des femmes aux élections provinciales de 2006 dans la ville de Kisangani(province orientale), thèse de doctorat en SPA, FSSAP/UNIKIS, 2013-2014

23. Wright M. (1988). « Autobiographies, histoires de vie et biographies de femmes africaines en tant que militants », Cahiers d'études africaines, vol. 28, n 109.

24. Y. Meni \& J.C Thoenig (1989). Politiques publiques, Paris PUF. 